\title{
Ad Hoc Reviewer Acknowledgment
}

The Editor, Dr. Steven Jay Lynn, along with his editorial staff, gratefully acknowledges the assistance of the following individuals, who served as ad hoc reviewers for Psychology of Consciousness: Theory, Research, and Practice between October 2015 and October 2016.

James Alcock

Fabrice Berna

Richard Block

Wayne Christensen

Liam Condon

Martin Conway

Aline Cordonnier

Romara Delmonte*

K. Andrew DeSoto

Joseph Glicksohn

Fallon R. Goodman

Oliver Hardt

Anne Hauswald

Simon Høffding

${ }^{*}$ Denotes co-reviewer.
Ralph Hood

James Houran

Michael Hyland

Harvey Irwin

Laura King

Karl Kuntzelman*

Rense Lange

Stephen Lankton

Miguel Marias

William Matthews

Reed Maxwell

Johnjoe McFadden

Christine Mohr

Bryan Myers
Henry Otgaar

Vince Polito

Anne Scharling

Rasmussen

Alan Scoboria

Madalina Sucala

Stefan Sutterlin

Connie Svob

Henry Szechtman

Emilie Thienot

Harald Walach

Bruce Wampold

Marc Wittmann

David Yaden 